\title{
Association between complementary feeding method and BMI at 19-36 months of life: an Internet-based cross-sectional study from two European countries
}

\author{
Monika A. Zielinska ${ }^{1}$, Petra Rust ${ }^{2}$, Jacqueline Bichler $^{2}$, Daria Masztalerz-Kozubek ${ }^{1}$ and \\ Jadwiga Hamulka ${ }^{1}$ \\ ${ }^{1}$ Faculty of Human Nutrition and Consumer Sciences, Warsaw University of Life Sciences (SGGW-WULS), Warsaw, \\ Poland and \\ ${ }^{2}$ University of Vienna, Department of Nutritional Sciences, Vienna, Austria
}

\begin{abstract}
Over the last years, there has been increasing interest in Baby-Led Weaning (BLW), an approach in which infants feed themselves with finger foods. Previous studies have shown that BLW may be associated with lower food fussiness and risk of obesity, but in the other hand with growth flattering. However, the results are limited and still inconclusive. The aim of this study was to investigate the associations between complementary feeding method and body mass index (BMI) z-score in children aged 19-36 months from Poland and Austria. This anonymously, the cross-sectional internet-based study was conducted in 2018 and 2019 among 6645 mothers of children aged 12-36 months, recruited through social media. The analysis included data from 5856 mothers (35.5\% from Austria) with complete data. Complementary feeding method was categorized as traditional spoon feeding (TSF), partial BLW (children was both spoon-fed and fed themselves), as well as BLW. BMI z-score was calculated based on declared body mass and height using the WHO cut-off points. BLW method was used in $16.4 \%$ of children, whereas the TSF in $30.5 \%$ of the total study group. Normal BMI z-score was observed in $73.8 \%$ of the total study group, thinness in $9.6 \%$ and overweight and obesity in $4.3 \%$. The BLW increased the risk of thinness only in children aged 31-36 months (aOR: 1.48; 95\%CI: 1.04-2.11) but did not influence the risk of overweight and obesity. In this present large cross-sectional study conducted in two European countries, we did not find an association between BLW and overweight and obesity risk. However, in the older group, BLW increased the risk of thinness. Previous studies observing a risk of thinness were also cross-sectional, although conducted among smaller study groups. In contrary to this, In contrary to this, the RCT trials using modified BLW version Baby-Led Introduction to SolidS (BLISS) did not observe any case of growth flattering. In another cross-sectional study investigating dietary intake of infants aged 6-8 months, who were complementary fed using BLW or TSF method, authors did not observe a difference in energy intake between the two groups. Further studies investigating the role of complementary feeding method and subsequent BMI, especially in long-term are necessary.
\end{abstract}

\section{Conflict of Interest}

There is no conflict of interest. 